\title{
Effects of Actuating Pressure Waveforms on the Droplet Behavior in a Piezoelectric Inkjet
}

\author{
Hsuan-Chung $\mathrm{Wu}^{1}$ and Huey-Jiuan $\mathrm{Lin}^{2}$ * \\ ${ }^{1}$ Department of Materials Engineering and Center for Thin Film Technologies and Applications, \\ Ming Chi University of Technology, Taipei, Taiwan, R. O. China \\ ${ }^{2}$ Department of Materials Science and Engineering, National United University, Miaoli, Taiwan, R. O. China
}

\begin{abstract}
This paper presents a numerical analysis of a piezoelectric actuated droplet generator. A three-dimensional finite difference numerical model based on a SOLA (solution algorithm) scheme for the solution of governing equations of the flow field and a volume-of-fluid method for tracing the fluid interfaces are presented. The surface tension is modeled using a continuum surface force concept and thus computed as a function of the interfacial curvature. The pressure pulse at the nozzle inlet, which is related to the applied voltage, was imposed according to the propagation theory of acoustic waves. The effects of the pressure waveforms, including positive and negative pressure amplitudes, operating period and acceleration of the positive pressure, on the droplet ejection process are simulated. The performance of piezoelectric inkjet: droplet break time, droplet tail length, droplet velocity and droplet volume are analyzed. The simulation results show that the tail length and volume of the droplet increase with the amplitude of the positive pressure and operating period. The breakup time of the droplet is shorter when the amplitude of the negative pressure increases. The factors that influence satellite droplets are also investigated in this study.
\end{abstract}

[doi:10.2320/matertrans.M2010123]

(Received April 9, 2010; Accepted October 6, 2010; Published November 17, 2010)

Keywords: inkjet, piezoelectricity, micro-droplet, pressure-waveform

\section{Introduction}

With the rapid development of inkjet technology, its application has been extended from conventional image printing to newer applications, such as Solder-Jet technology, ${ }^{1)}$ color filter in flat panel display, ${ }^{2)}$ solar cells, ${ }^{3)}$ sensors, ${ }^{4)}$ nano-powder suspension patterning ${ }^{5)}$ and various material processes. ${ }^{6)}$ The attractive features of this technology are non-contact, reduced material wastage, mask-less, low cost, ability to large area manufacturing and reduction of environmental impact.

There are several types of inkjet heads to eject micro droplets, including piezoelectric, thermal bubble, electrostatic and acoustic methods. ${ }^{7)}$ Among them, piezoelectric and thermal bubble actuation methods are the most mature and popular systems for commercial inkjet printers. The ejection of droplets from the nozzle is induced either by the displacement of a piezoelectric diaphragm that is in contact with the fluid or by the formation of a vapor bubble in the ink through the heating of a resistive film. Since piezoelectric inkjet printing systems have no need to vaporize the fluid, they can be used for the ejection and dispensing of polymer and metal inks.

The printing quality is closely related to the properties of ejected droplet, such as the velocity and shape fluctuations of the droplets, droplet size, as well as the satellite drops. Without detailed knowledge of the pressure response and velocity variation in the ink flow channel, optimal droplet ejection and therefore optimal printing quality cannot be assured.

In order to look deeply into the phenomena of droplet ejected process and accelerate the relevant technology, numerical simulation was employed by many researchers.

*Corresponding author, E-mail: hjlin@nuu.edu.tw
Before simulation, it is necessary to derive the pressure history at the nozzle end of the chamber due to the expansion and contraction of the chamber walls resulting from the application of a voltage pulse to the piezoelectric tube. The response of the piezoelectric crystal tube itself was studied in Bugdayci et al., ${ }^{8)}$ in which the inner cylindrical wall displacement of the tube was calculated for an applied voltage. Bogy and Talke ${ }^{9)}$ calculated the variation of pressure at the nozzle inlet, which was caused by the expansion and contraction of the piezoelectric tube. A numerical method for the drop formation based on the axisymmetric Navier-Stokes equations was presented by Fromm. ${ }^{10)}$ The impact of square wave pressure applied at a nozzle inlet on drop velocities and structure of ejected drops was discussed. Liou et al. ${ }^{11)}$ developed a fluid dynamics simulation system based on a finite volume scheme and a volume-of-fluid method to predict the fluid interface. The numerical prediction of the meniscus position related to pressure plate motion inside the nozzle during the infusion and ejection stages were presented. Yang et al. ${ }^{12)}$ investaged the droplet ejection process for a Picojet print-head by the commercial software CFD-ACE + and examined the droplet quality based on 17 numerical experiments, including exit diameter of nozzle, ejection time and the fluid's physical properties. Sen et al. ${ }^{13)}$ presented a simulation study of the droplet ejection performance of a thermal inkjet print-head. Effects of print head geometry, operating conditions, and properties of the ink on the performance of the inkjet device were investigated. Kim et al. ${ }^{14)}$ performed a conceptual design using computational fluid dynamics (CFD) and micro-electro-mechanical systems (MEMS) fabrication to develop an industrial inkjet head for micro-patterning on printed circuit boards. The measured displacement waveform from a piezoelectric actuator by laser doppler vibrometer (LDV) was used as input data for the three dimensional flow solver to simulate the droplet 
formation. In our previous studies, ${ }^{15-17)}$ a computer-aided analysis system was developed to simulate the formation, ejection, and impact of the droplet on the substrate for a piezoelectric inkjet printing device. The numerical model was also validated by comparing the simulated results with experimental observations/measurements. The simulated results are rather consistent with the experimental observations in terms of droplet morphology, break-up time, flying distance, and droplet volume. We also studied the effects of ink properties including viscosity and surface tension and operational characteristics including pressure wave shape and cavity length on the formation of liquid droplets.

According to the literatures mentioned, the flow behavior of inkjet printing process depends strongly on print-head design, operating parameters and fluid properties. The parametric studies have been performed by mathematical model or experimental observation in many previous works. To authors' knowledge, few of literature are focused on how the actuating pressure wave affects the droplet behavior in ejection process. Therefore, the purpose of this study is to present numerical experimental results for a piezoelectric inkjet printing process and study the effects of the individual component of the actuating pressure wave conditions, including the positive and negative amplitudes of pressure wave, operating time, and acceleration of positive pressure on the formation and flying of liquid droplet. The quality of droplets is evaluated by indices of the satellites separating from a main droplet, the shape and volume as well as velocity of droplets during ejection.

\section{Mathematical Model}

Because of the complexities involved, transient initial value problems and finite deformation of a liquid-gas interface, which describe a droplet ejection process, the numerical calculation should then be capable of dealing with the free surface flow, tracking the interface location, imposing the effects of surface tension and handling the complicated boundary conditions. In this study, a threedimensional numerical approach comprising of the VolumeOf-Fluid $(\mathrm{VOF})^{18)}$ method for tracking the interface location and the Continuum Surface Force (CSF) ${ }^{19)}$ method for modeling the effects of surface tension is employed.

\subsection{Governing equations}

The most inks used in inkjet printing are dilute polymer solutions in water. Their viscosity is a weak function of shear rate and consequently can be approximated by a Newtonian (constant viscosity) fluid. Like most liquids these inks are also incompressible. The equations of motion can be written in the form of the Navier-Stokes equations as following.

$$
\begin{aligned}
\frac{\partial \vec{V}}{\partial t} & +(\vec{V} \cdot \nabla) \vec{V} \\
& =-\frac{1}{\rho} \nabla P+\frac{\mu}{\rho} \nabla \cdot\left(\nabla \vec{V}+\nabla \vec{V}^{T}\right)+\vec{g}+\frac{1}{\rho} \vec{F}_{\mathrm{b}}
\end{aligned}
$$

Where $\rho\left(\mathrm{kg} \cdot \mathrm{m}^{-3}\right)$ is density; $\mu(\mathrm{Pa} \cdot \mathrm{s})$ is viscosity; $P(\mathrm{~Pa})$ is pressure field; $\vec{V}\left(\mathrm{~m} \cdot \mathrm{s}^{-1}\right)$ is the velocity field; $\vec{F}_{\mathrm{b}}(\mathrm{N})$ is the body force; $\vec{g}\left(\mathrm{~m} \cdot \mathrm{s}^{-2}\right)$ is the acceleration due to gravity.
The velocity $\vec{V}$ is constrained by the continuity equation (conservation of mass), which, for an incompressible flow, takes the form

$$
\nabla \cdot \vec{V}=0
$$

\subsection{Treatments for the free surface flow}

Three crucial problems in modeling free surface flows are how to estimate the free surface location, monitor the evolution of the fluid domain and handle the free surface boundary conditions. In this study, a Volume-of-Fluid (VOF) interface tracking method, which was developed by Nichols and Hirt, ${ }^{18)}$ is adapted to represent the fluid domain and to track the evolution of its free boundaries.

With the VOF method, a field variable, $F(x, y, z, t)$, is designated to each computational element to indicate the volume fraction of liquid in that particular cell. When $F$ is equal to 1 , it means the cell is full of liquid. When $F$ is equal to 0 , it means the cell is full of gas (or empty of liquid). When $F$ is between 0 and 1, the element contains both liquid and gas and an interface is then allocated in that particular cell. Thus, the $F$ value can indicate the domain of fluid flow and it is a step function. From the law of mass conservation, the volume fraction of fluid, $F$, is governed by the following equation:

$$
\frac{\partial F}{\partial t}+(\vec{V} \cdot \nabla) F=0
$$

As the flow field $(u, v, w)$ is known, it can update $F$ according to eq. (3). Thus, it can get new configuration and distribution of fluid. However, if this equation is solved by using regular finite difference scheme, $F$ is soon smeared due to numerical diffusion and interfaces are no longer sharp. Furthermore, liquid volume may not be conserved exactly and therefore, must be treated by other methods.

When dealing with the advection of $F$, the interface in a surface cell must be constructed to provide the correct $F$ value transported. A number of techniques have been proposed to maintain a well defined interface within the volume fraction framework. The well known SLIC (Simple Line Interface Calculation) method ${ }^{20)}$ was developed to deal with the interface position in earlier works. The original SLIC method approximates the interface in each mesh cell as a line parallel to one of the coordinate axes in a twodimensional analysis. The interface representation using SLIC is obviously very rough. However, it has been adopted in many problems, especially when less deformation was involved. The PLIC (Piecewise Linear Interface Calculation) method, which was developed by Youngs, ${ }^{21)}$ gave a useful refinement to the SLIC method with the use of a straight line to approximate the interface within each computational cell. The direction of the straight line is determined by the liquid volumes in that particular cell and its neighboring cells.

The PLIC ${ }^{22-24)}$ method is adopted and then coupled with the VOF method in this study. It approximates the interface by a plane, where the orientation is properly determined, within each computational cell. Once the interface is constructed or reconstructed, its motion by the velocity field must be modeled by a suitable advection algorithm. This can be achieved by either an Eulerian or a Lagrangian scheme. 
The Lagrangian scheme, which computes directly the motion of the interface, is adopted in this study. Then, with the displacement of the plane, the advection of $F$ can be calculated. The numerical scheme consists of the following three steps:

Step 1: Construct/Reconstruct interface with planes.

Step 2: Solve for the velocity field.

Step 3: Update $F$.

After the $F$ values of all the cells are determined, a normal direction to the interface for each surface cell is estimated by the following equation:

$$
\vec{n}=\nabla F
$$

The interface of a surface cell can then be represented by the following equation for a plane:

$$
-\vec{x} \cdot \hat{n}-\alpha=0
$$

Where $\alpha$ is a plane constant; $\vec{x}$ is a position vector; $\hat{n}$ is the unit normal vector of the plane. The negative sign indicates that the direction of the unit vector points towards the air. A $3 \mathrm{D}$ inverse technique employed by Scardovelli ${ }^{23)}$ can obtain the analytical solution of a through a sequence of simple linear geometrical transformations, translations, and mirror reflections. It is computationally cheaper than obtaining the value by solving a set of simultaneous equations.

After step 2, the flow field $\left(u^{*}, v^{*}, w^{*}\right)$ at the new time step, $t^{*}(t+\Delta t)$, is known. At each cell face with a velocity of $u^{*}$, $v^{*}$ or $w^{*}$, a layer of fluid, the thickness of which is approximately equal to $u^{*} \Delta t, v^{*} \Delta t$ or $w^{*} \Delta t$, is transported from the upstream to the downstream cell during the time interval of $\left[t, t^{*}\right]$. The transported liquid volume in this layer, $V_{\text {adv }}$, is determined geometrically by using the reconstructed interface of the old time $t$ (Step 1). For each cell, three contributions; $\left(\phi^{-}\right)_{i, j, k},\left(\phi^{+}\right)_{i, j, k}$ and $\left(\phi^{0}\right)_{i, j, k}$, are calculated. $\left(\phi^{-}\right)_{i, j, k}$ and $\left(\phi^{+}\right)_{i, j, k}$ represent the volume fluxes, which enter the $(i-1, j, k)$ and $(i+1, j, k)$ cells, respectively, from the $(i, j, k)$ cell. $\left(\phi^{0}\right)_{i, j, k}$ is the remained fluid volume in the $(i, j, k)$ cell. If the fluid is flowing out of the cell through the right boundary then $\left(\phi^{+}\right)_{i, j, k}$ is larger than 0 and $\left(\phi^{-}\right)_{i+1, j, k}$ is equal to 0 . If fluid flows through the left boundary, then $\left(\phi^{-}\right)_{i, j, k}$ is larger than 0 and $\left(\phi^{+}\right)_{i-1, j, k}$ is equal to 0 . The three volumes are the regions under the advected line interfaces, which cut through the $(i, j, k)$ cell as shown in Fig. 1. The updated volume fraction in each cell after the fractional step along the $x$ direction is then given by

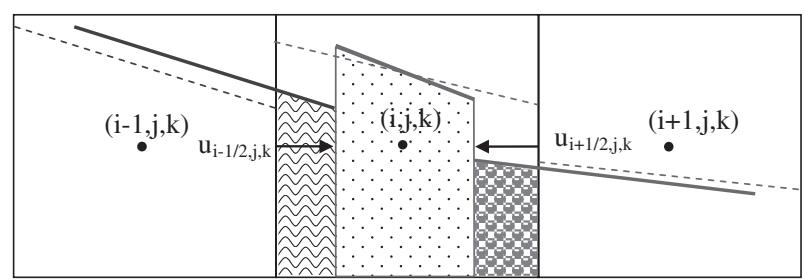

$$
\begin{aligned}
& \left(\phi^{+}\right)_{\mathrm{i}-1, \mathrm{j}, \mathrm{k}} \quad\left(\phi^{0}\right)_{\mathrm{i}, \mathrm{j}, \mathrm{k}} \quad\left(\phi^{-}\right)_{\mathrm{i}+1, \mathrm{j}, \mathrm{k}} \\
& \text { _ } \text { segment after advection } \\
& \text { - } \text { segment before advection } \\
& \text { cell boundaries }
\end{aligned}
$$

Fig. 1 Calculation of the volume fluxes during one fractional step.

$$
F_{i, j, k}^{(*, x)}=\left(\phi^{-}\right)_{i+1, j, k}+\left(\phi^{0}\right)_{i, j, k}+\left(\phi^{+}\right)_{i-1, j, k}
$$

The treatment of the updated volume fraction in each cell after the fractional time step along the $y$ and $z$ directions are also then given by

$$
\begin{aligned}
& F_{i, j, k}^{(*, y)}=\left(\phi^{-}\right)_{i, j+1, k}+\left(\phi^{0}\right)_{i, j, k}+\left(\phi^{+}\right)_{i, j-1, k} \\
& F_{i, j, k}^{(*, z)}=\left(\phi^{-}\right)_{i, j, k+1}+\left(\phi^{0}\right)_{i, j, k}+\left(\phi^{+}\right)_{i, j, k-1}
\end{aligned}
$$

As the $F$ values in the three axial coordinates are calculated, a new distribution of the $F$ values can then be obtained.

\subsection{Implementation of surface tension treatments for the free surface flow}

For the fluid flow problems of droplet formation/ejection, surface tension is the dominant force at the interface. In order to take the effects of surface tension into consideration, special boundary conditions must be applied to the NavierStokes equations at the interfaces. However, this approach suffers from the difficulties in modeling topologically complex interfaces having surface tension. Brackbill ${ }^{19)}$ developed a very different approach to deal with the issue of surface force. It is called the CSF (Continuum Surface Force) method. CSF method converts the interfacial surface force into a corresponding volume force, which can then be included in the Navier-Stokes equations. The volume force, nonzero only at a free surface, is given by

$$
\vec{F}_{\mathrm{b}}=\sigma \kappa \nabla F
$$

where $\sigma\left(\mathrm{N} \cdot \mathrm{m}^{-1}\right)$ is the surface tension of the fluid-gas interface, $\kappa\left(\mathrm{m}^{-1}\right)$ is the local free surface curvature and calculated by eq. (10)

$$
\kappa=-\left(\nabla \cdot \frac{\vec{n}}{|\vec{n}|}\right)
$$

With the volume force, surface tension effects at free surfaces are modeled as a body force in the momentum transport eq. (1).

\section{Results and Discussion}

The geometry and computational domain is shown in Fig. 2 with the related sizes depicted. A simplified coneshape nozzle is employed. The nozzle exit is $40 \mu \mathrm{m}$ in diameter. The flying distance from nozzle to substrate is $800 \mu \mathrm{m}$. With deionized water as ink, the related density, viscosity and surface tension are $1000 \mathrm{~kg} / \mathrm{m}^{3}, 0.001 \mathrm{~Pa} \cdot \mathrm{s}$ and $0.072 \mathrm{~N} / \mathrm{m}$ respectively. Because the density and viscosity of the ambient gas are so small compared to the liquid, there is less effect on the simulation results. Thus, the ambient gas is treated as a void (zero density) in the simulation system. From preliminary tests, the maximum of liquid velocity is $8 \mathrm{~m} / \mathrm{s}$ and the maximum of the Reynolds number is about 320. Thus, the fluid is treated as laminar flow.

The principle of operation of the piezoelectric inkjet print head is based on the acoustic wave theory. The electrically induced motion of the piezoelectric crystal compresses the ink in contact with the transducer, forming an acoustic pressure pulse. ${ }^{8)}$ The pressure pulse interacts with the fluid down to the nozzle. The pressure waveform can be changed by applying different voltage pulse form. The experimental 


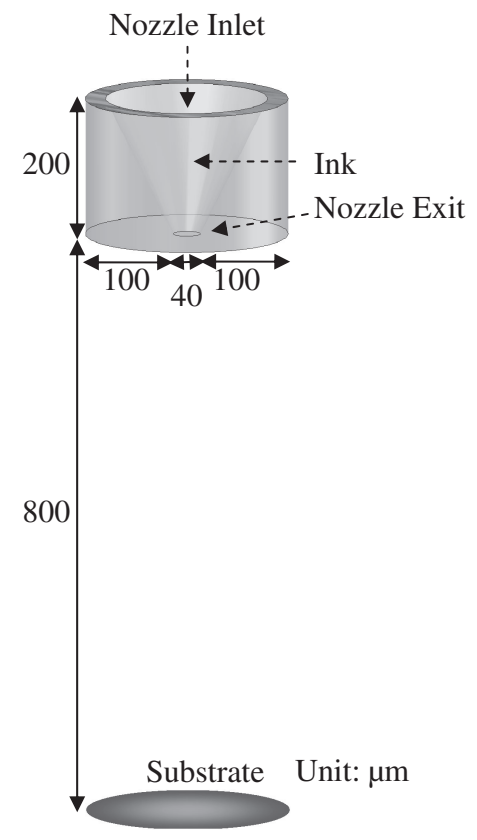

Fig. 2 A schematic diagram of the inkjet print-head showing the nozzle configuration and dimensions of the modeled system.

observation of the droplet ejection behaviors caused by a single or a double voltage pulse is successfully explained through the propagation of acoustic pressure waves. ${ }^{25,26)}$ During positive pressure period, the meniscus at the nozzle exit is formed and then a jet is created. The ejected liquid is separated from nozzle when the negative pressure is applied.

The pressure waveform at the nozzle inlet, as shown in Fig. 3, is imposed in this study to discuss the effects of pressure parameters on the droplet ejection. The positive pressure is imposed in the duration of 0 to $t_{\mathrm{p}}$, and the negative pressure wave is imposed during $t_{\mathrm{p}}$ to $t_{\mathrm{n}}$. The breakup of droplet is most occurred in the period of $t_{\mathrm{p}}$ to $t_{\mathrm{n}}$. No more pressure wave is applied after $t_{\mathrm{n}}$. The pressure pulse parameters tested in this work are amplitude, operating time and accelerated rate, as shown in Table 1. The baseline is as the standard case and compares with the other 8 cases. The influence of (1) the positive pressure amplitude $\left(P_{\max }\right),(2)$ the negative pressure amplitude $\left(P_{\min }\right),(3)$ the total operating time $\left(t_{\mathrm{o}}=t_{\mathrm{r} 1}+t_{\mathrm{f} 1}+t_{\mathrm{r} 2}+t_{\mathrm{f} 2}\right)$, (4) acceleration of the positive pressure $\left(a_{\mathrm{p}}=P_{\max } / t_{\mathrm{r} 1}\right)$ on ejection behavior are

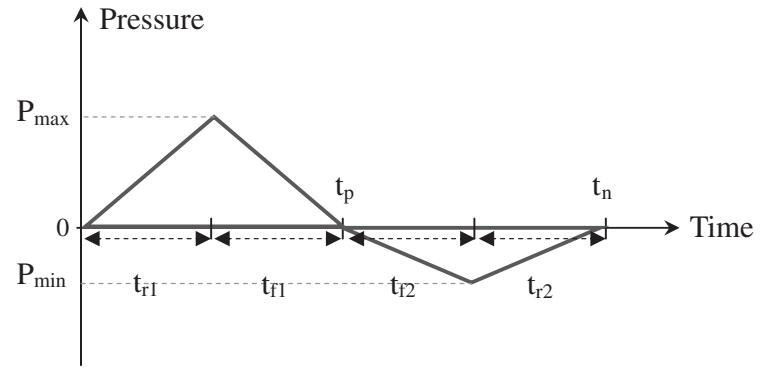

Fig. 3 Pressure-time relations at nozzle inlet.

discussed. The $a_{\mathrm{p}}$ is defined as 1 for the baseline. Therefore, the $a_{\mathrm{p}}$ values for the case 7 and case 8 are 2 and $2 / 3$ respectively.

Three different mesh systems, $48 \times 48 \times 200,60 \times 60 \times 250$ and $72 \times 72 \times 300$, were evaluated for the baseline as shown in Figs. 4-6. During droplet formation period (at 16 and $32 \mu \mathrm{s}$ ), the droplet shapes and velocities of these three systems are almost the same. From 54 to $102 \mu \mathrm{s}$, the droplet shapes and velocities of the $60 \times 60 \times 250$ and $72 \times 72 \times 300$ systems are consistence, but are different from the $48 \times 48 \times 200$ system. After $138 \mu \mathrm{s}$, the velocity of the satellite droplet of the $60 \times 60 \times 250$ system is different from the $72 \times 72 \times 300$ system, but the droplet shape and velocity of the main droplet are consistence. Therefore, the simulation system is divided into a uniform as well as rectangular mesh system of $60 \times 60 \times 250$. The simulated results are shown in Figs. 7-14.

The cross-section views at center plane of modeling area are presented by a sequence of pictures to show the droplet morphology and velocity contour. From a close examination of the droplet ejection sequences, it can be divided into two stages, droplet formation process and droplet flying process. During droplet formation period, the ink is pushed out and the meniscus forms at nozzle exit. After breakup from nozzle exit, the droplet changes shape, flies and hits on the substrate during flying sequences. The pictures (a)-(c) in each figure show three sequences of drop formation at time instants of $0.5 t_{\mathrm{p}}(\mathrm{a}), t_{\mathrm{p}}(\mathrm{b})$ and breakup time (c). The pictures (d) $-(\mathrm{g})$ in each figure show the sequences of drop separating from the nozzle exit, flying and hitting on substrate. The picture (d) is at time instant of stopping applied pressure (time $=t_{\mathrm{n}}$ ), and $(\mathrm{g})$ is at time when drop hits on substrate. The pictures of (e) and (f) are the flying instants chosen at equi-interval between (d) and (g).

Table 1 Simulation conditions for actuating pressure wave.

\begin{tabular}{|c|c|c|c|c|c|c|c|}
\hline & $\begin{array}{c}P_{\max } \\
(\mathbf{M P a})\end{array}$ & $\begin{array}{c}P_{\min } \\
(\mathbf{M P a})\end{array}$ & $\begin{array}{c}t_{\mathrm{r} 1} \\
(\mu s)\end{array}$ & $\begin{array}{c}t_{\mathrm{f} 1} \\
(\mu s)\end{array}$ & $\begin{array}{c}t_{\mathrm{r} 2} \\
(\mu s)\end{array}$ & $\begin{array}{c}t_{\mathrm{f} 2} \\
(\mu \mathrm{s})\end{array}$ & Figure \\
\hline Baseline & 0.05 & 0.025 & 16 & 16 & 16 & 16 & Fig. 5 \\
\hline Case 1 & 0.03 & 0.025 & 16 & 16 & 16 & 16 & Fig. 7 \\
\hline Case 2 & 0.07 & 0.025 & 16 & 16 & 16 & 16 & Fig. 8 \\
\hline Case 3 & 0.05 & 0.01 & 16 & 16 & 16 & 16 & Fig. 9 \\
\hline Case 4 & 0.05 & 0.04 & 16 & 16 & 16 & 16 & Fig. 10 \\
\hline Case 6 & 0.05 & 0.025 & 8 & 8 & 8 & 8 & Fig. 12 \\
\hline Case 7 & 0.05 & 0.025 & 8 & 24 & 16 & 16 & Fig. 13 \\
\hline Case 8 & 0.05 & 0.025 & 24 & 8 & 16 & 16 & Fig. 14 \\
\hline
\end{tabular}




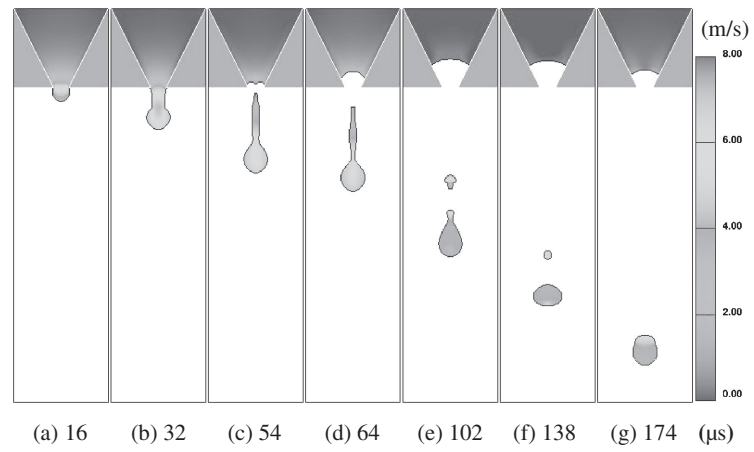

Fig. 4 Evolution of droplet shape and velocity contour for the baseline with mesh system of $48 \times 48 \times 200, P_{\max }=0.05 \mathrm{MPa}, P_{\min }=0.025 \mathrm{MPa}$, $t_{\mathrm{o}}=64 \mu \mathrm{s}$ and $a_{\mathrm{p}}=1$.

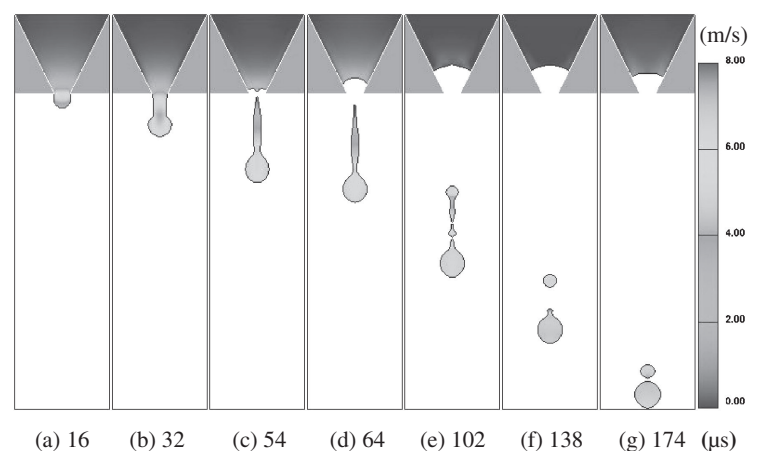

Fig. 5 Evolution of droplet shape and velocity contour for the baseline with mesh system of $60 \times 60 \times 250, P_{\max }=0.05 \mathrm{MPa}, P_{\min }=0.025 \mathrm{MPa}$, $t_{\mathrm{o}}=64 \mu \mathrm{s}$ and $a_{\mathrm{p}}=1$.

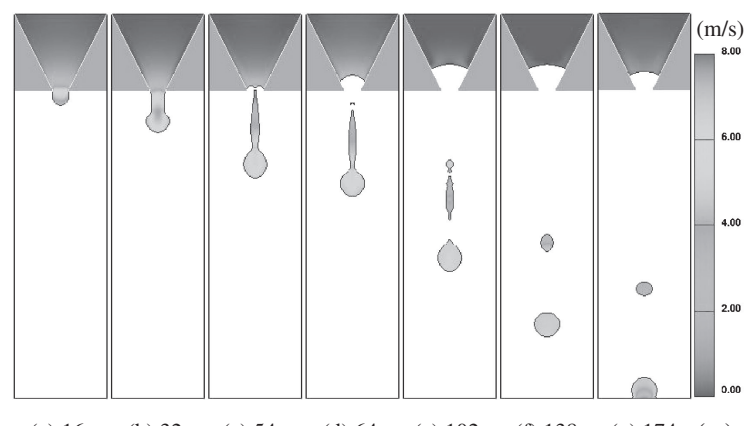

$\begin{array}{lllllll}\text { (a) } 16 & \text { (b) } 32 & \text { (c) } 54 & \text { (d) } 64 & \text { (e) } 102 & \text { (f) } 138 & \text { (g) } 174\end{array}$

Fig. 6 Evolution of droplet shape and velocity contour for the baseline with mesh system of $72 \times 72 \times 300, P_{\max }=0.05 \mathrm{MPa}, P_{\min }=0.025 \mathrm{MPa}$, $t_{\mathrm{o}}=64 \mu \mathrm{s}$ and $a_{\mathrm{p}}=1$.

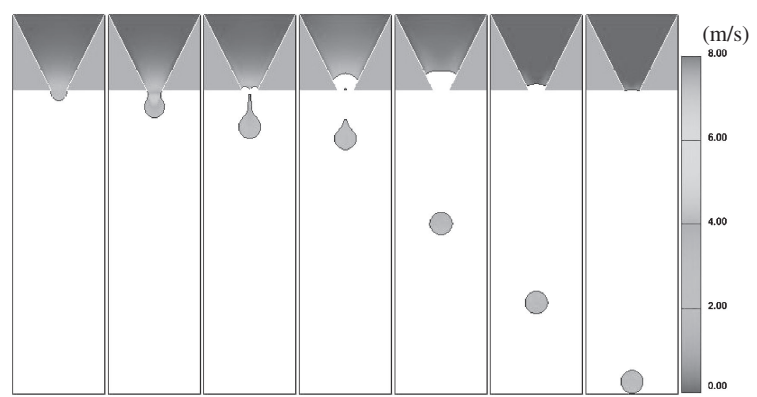

$\begin{array}{lllllll}\text { (a) } 16 & \text { (b) } 32 & \text { (c) } 52 & \text { (d) } 64 & \text { (e) } 182 & \text { (f) } 300 & \text { (g) } 416 \quad \text { ( } \mu \text { s) }\end{array}$

Fig. 7 Evolution of droplet shape and velocity contour for case 1 with $P_{\max }=0.03 \mathrm{MPa}, P_{\min }=0.025 \mathrm{MPa}, t_{\mathrm{o}}=64 \mu \mathrm{s}$ and $a_{\mathrm{p}}=1$.

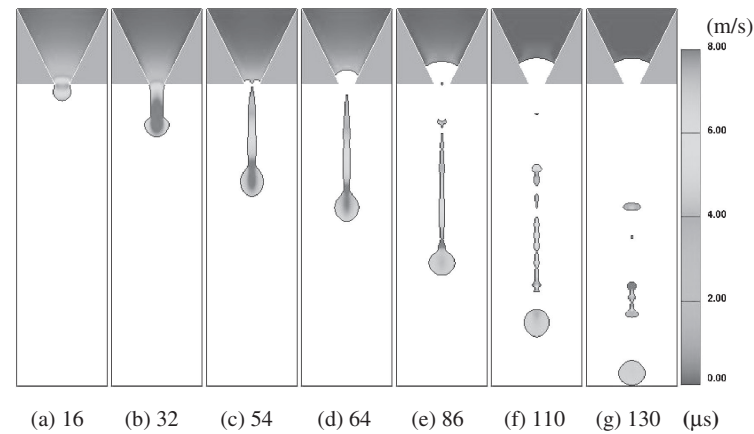

Fig. 8 Evolution of droplet shape and velocity contour for case 2 with $P_{\max }=0.07 \mathrm{MPa}, P_{\min }=0.025 \mathrm{MPa}, t_{\mathrm{o}}=64 \mu \mathrm{s}$ and $a_{\mathrm{p}}=1$.

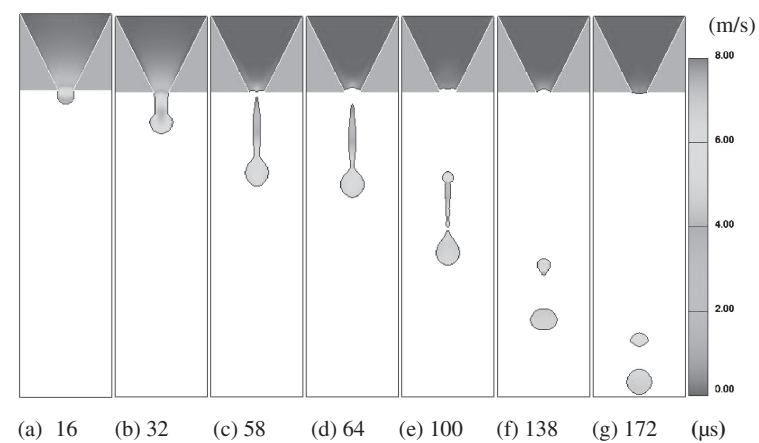

Fig. 9 Evolution of droplet shape and velocity contour for case 3 with $P_{\max }=0.05 \mathrm{MPa}, P_{\min }=0.01 \mathrm{MPa}, t_{\mathrm{o}}=64 \mu \mathrm{s}$ and $a_{\mathrm{p}}=1$.

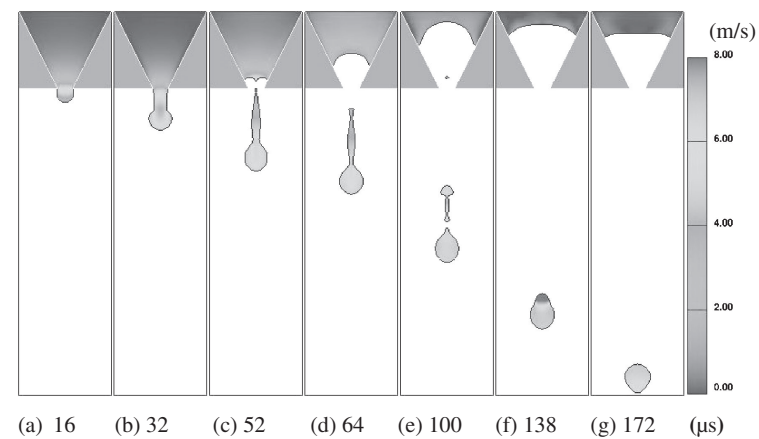

Fig. 10 Evolution of droplet shape and velocity contour for case 4 with $P_{\max }=0.05 \mathrm{MPa}, P_{\min }=0.04 \mathrm{MPa}, t_{\mathrm{o}}=64 \mu \mathrm{s}$ and $a_{\mathrm{p}}=1$.

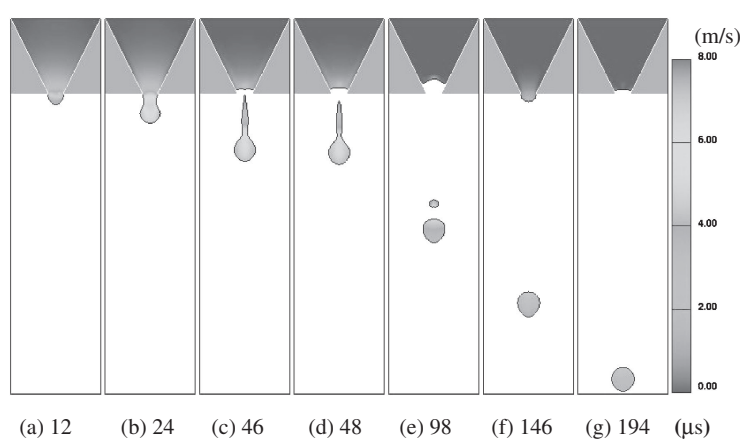

Fig. 11 Evolution of droplet shape and velocity contour for case 5 with $P_{\max }=0.05 \mathrm{MPa}, P_{\min }=0.025 \mathrm{MPa}, t_{\mathrm{o}}=48 \mu$ s and $a_{\mathrm{p}}=1$. 


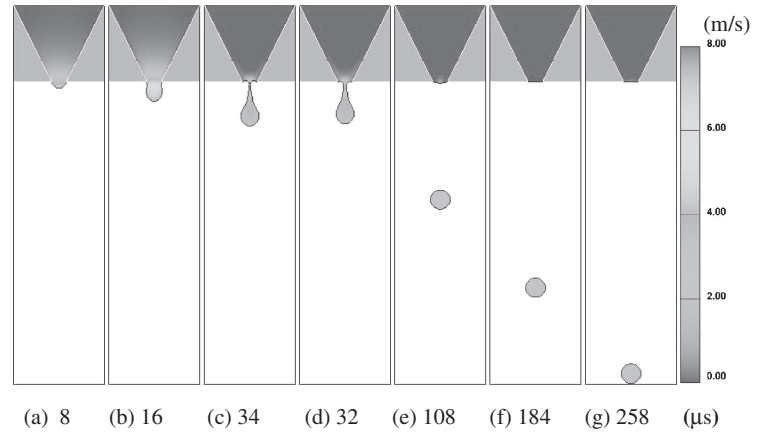

Fig. 12 Evolution of droplet shape and velocity contour for case 6 with $P_{\text {max }}=0.05 \mathrm{MPa}, P_{\text {min }}=0.025 \mathrm{MPa}, t_{\mathrm{o}}=32 \mu \mathrm{s}$ and $a_{\mathrm{p}}=1$.

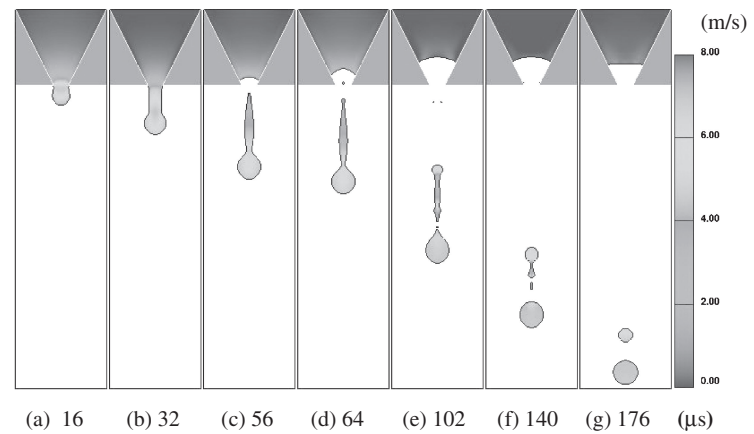

Fig. 13 Evolution of droplet shape and velocity contour for case 7 with $P_{\text {max }}=0.05 \mathrm{MPa}, P_{\text {min }}=0.025 \mathrm{MPa}, t_{\mathrm{o}}=64 \mu \mathrm{s}$ and $a_{\mathrm{p}}=2$.

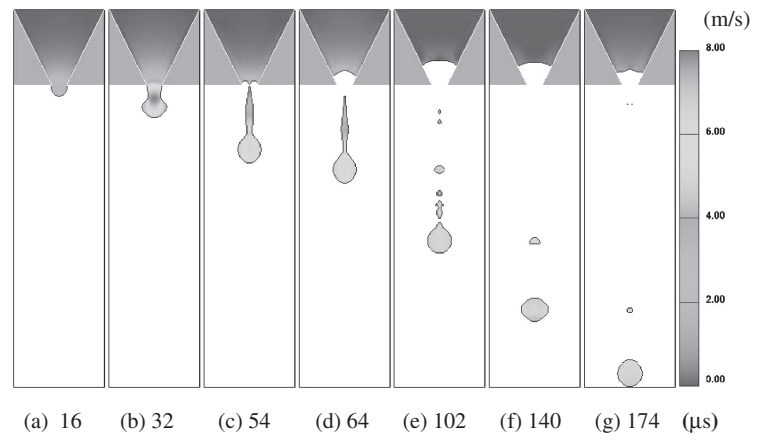

Fig. 14 Evolution of droplet shape and velocity contour for case 8 with $P_{\text {max }}=0.05 \mathrm{MPa}, P_{\min }=0.025 \mathrm{MPa}, t_{\mathrm{o}}=64 \mu$ s and $a_{\mathrm{p}}=2 / 3$.

\subsection{Droplet formation process}

\subsubsection{Droplet formation process of the baseline case}

Time evolution of the droplet shape together with velocity contours in the fluid is depicted in Fig. 5 for the baseline case. As time progresses after the pressure is applied, the liquid ejected out of nozzle exit gradually changes its shape from a meniscus to a teat-shape droplet. In the period of $t_{\mathrm{r} 1}$ from 0 to $16 \mu \mathrm{s}$, the accelerated positive pressure is applied on the inlet of nozzle and the liquid is ejected at the exit of nozzle. The shape of the liquid is like a meniscus as shown in Fig. 5(a). In $t_{\mathrm{f} 1}$ from 16 to $32 \mu \mathrm{s}$, the positive pressure reduces from $P_{\max }$ to 0 , still keeps exerting its effect on the liquid, and a droplet with column tail forms, as shown in Fig. 5(b). This shape is formed because the ejected liquid has a higher velocity at the drop tip than that at the nozzle exit. In the time period of 32 to $64 \mu \mathrm{s}\left(t_{\mathrm{r} 2}+t_{\mathrm{f} 2}\right)$, the negative pressure is applied and the liquid near the exit is affected by both the upward pulling force and downward inertia force. Then, necking of the ejected liquid occurs at the tip of the nozzle. At around $54 \mu \mathrm{s}$ in this case, the liquid's forward inertia momentum overcomes the opposed effects of surface tension and viscous force, and a droplet breakup is observed with a tailing droplet, as shown in Fig. 5(c). In other words, the ejected ink separates from the nozzle exit. During the droplet flying process (Fig. 5(d)-(g)), the droplet undergoes shape fluctuation and reaches the print media.

\subsubsection{Influence of $\boldsymbol{P}_{\max }$ on the droplet formation process}

The effect of the positive pressure amplitude $P_{\max }$ on the droplet formation is illustrated in pictures (a)-(c) of Figs. 7, 5 and 8 with $P_{\max }=0.03,0.05$, and $0.07 \mathrm{MPa}$, respectively. It is found that the breakup time is 52,54 and $54 \mu \mathrm{s}$, and the corresponding length of the broken liquid column is 128, 224 and $296 \mu \mathrm{m}$. The breakup time is not changed obviously with the positive pulse amplitude $P_{\max }$ under the same $t_{\mathrm{p}}, t_{\mathrm{n}}$ and $P_{\min }$. However, the tail length and velocity of droplet increases with an increase in $P_{\max }$. Tail length and velocity increase with applied pressure due to the fact that at higher pressure amplitude, a larger force acts on the ink in the nozzle and therefore drop velocity increases.

3.1.3 Influence of $\boldsymbol{P}_{\text {min }}$ on the droplet formation process The pictures (a)-(c) in Figs. 9 and 10 present the droplet formation for $P_{\min }=0.01$ and $0.04 \mathrm{MPa}$ at constant other parameters $\left(P_{\max }=0.05, t_{\mathrm{o}}=64 \mu \mathrm{s}\right.$ and $\left.a_{\mathrm{p}}=1\right)$. Comparing with Fig. 5(a)-(c), it is found that the breakup time decreases from 58, 54 to $52 \mu \mathrm{s}$ when the $P_{\min }$ increases from $P_{\text {min }}=0.01,0.025$ to $0.04 \mathrm{MPa}$, and the corresponding lengths of the broken liquid column are 244, 224 and $216 \mu \mathrm{m}$, respectively. The amplitude of the negative pressure affects the breakup time of the liquid column. The lager $P_{\min }$ induces larger upward pulling force and results in the tendency of the shorter breakup time and the shorter length of the liquid column. No apparent difference is observed in the drop velocity with changes in $P_{\min }$.

\subsubsection{Influence of $\boldsymbol{t}_{\mathrm{o}}$ on the droplet formation process}

The pictures (a)-(c) in Figs. 11 and 12 show the sequences of droplet formation at pulse length $t_{\mathrm{o}}=48$ and $32 \mu \mathrm{s}$. Comparing with Fig. 5(a)-(c), it is found that the breakup time decreases from 54,46 to $34 \mu \mathrm{s}$ when the total operating time decreases from $t_{\mathrm{o}}=64,48$ to $32 \mu \mathrm{s}$, and the corresponding lengths of the broken liquid column are 224, 180 and $120 \mu \mathrm{m}$, respectively. The length of droplet tail increases with $t_{\mathrm{o}}$ because of the increasing of the width of the positive pressure. Mostly, the breakup time of droplet occurs in the period of $t_{\mathrm{p}}-t_{\mathrm{n}}$, negative pressure applying. However, in the case 6 , the droplet separates from nozzle exit at $34 \mu \mathrm{s}$, longer than $t_{\mathrm{n}}(=32 \mu \mathrm{s})$ as shown in Fig. 12(c) $-(\mathrm{d})$.

\subsubsection{Influence of $\boldsymbol{a}_{\mathrm{p}}$ on the droplet formation process}

Pictures (a)-(c) in Figs. 5, 13 and 14 show the sequences of droplet formation for $a_{\mathrm{p}}=1,2$ and 2/3. Comparing pictures (a) and (b) in Figs. 5, 13 and 14, it is observed that the velocity in Fig. $11\left(a_{\mathrm{p}}=2\right)$ is highest at $16 \mu$ s and slowest at $32 \mu$ s than others because of the positive pressure reaching maximum value in shortest time $\left(t_{\mathrm{r} 1}=8 \mu \mathrm{s}\right)$, and the smallest positive pressure during $16-32 \mu \mathrm{s}$. As seen in the simulation 
results in Figs. 13, 5 and 14, the length of the broken liquid column is 248,224 and $204 \mu \mathrm{m}$, respectively, decreases with a decrease in $a_{\mathrm{p}}$. This shows that accelerating pressure in shorter time pushes out more ink, and the length of the broken liquid column becomes longer. The breakup time of droplet is 56, 54 and $54 \mu \mathrm{s}$ for each case of $a_{\mathrm{p}}=2,1$ and 2/3. The effect of $a_{\mathrm{p}}$ on breakup time is not obvious.

\subsection{Droplet flying process}

\subsubsection{Droplet flying process of the baseline}

When the liquid is completely separated from the nozzle exit, the finial droplet shape is determined by the droplet configuration and velocity distribution at the breakup instant. The droplet changes from a cylindrical column to a tear-like shape when it separates from nozzle exit as shown in Fig. 5(c). The ink in the nozzle is drawn back because of negative pressure. The tail is then pulled into the primary droplet due to the surface tension and viscous force during flying process. If the forward inertia momentum of primary droplet overcomes the opposed effect of surface tension and viscous force, a neck between tail and primary droplet occurs as shown in Fig. 5(d)-(e). Then a satellite droplet is formed and separates from the primary droplet. The velocity of the satellite droplet is higher than that of the main droplet and their distance becomes more closely, as shown in Fig. 5(f)-(g).

\subsubsection{Influence of $\boldsymbol{P}_{\max }$ on the droplet flying process}

Figures 7 and 8 show the time evolutions of droplet ejection at difference pressure amplitude $P_{\max }$. Compared with baseline pressure amplitude of $P_{\max }=0.05 \mathrm{MPa}$ (Fig. 5), smaller pressure amplitude of $0.03 \mathrm{MPa}$ (Fig. 7) could direct a less amount of liquid mass toward the nozzle, leading to a lower ejection velocity, thus reduce the length of droplet tail. Because of surface tension, the droplet becomes round and converge into a single, spherical shape in a state of minimum energy (as shown in Fig. 7(d)-(g)). In contrast, more liquid mass would flow through nozzle with a higher ejection velocity for a larger pressure amplitude such as $P_{\text {max }}=0.07 \mathrm{MPa}$ (as shown in Fig. $8(\mathrm{~d})-(\mathrm{g})$ ), and produces an elongated liquid strand in succession. Under such circumstances, multiple breakups of liquid are expected with the appearance of satellite droplets in the flying process. The average droplet velocity before arriving the substrate increase from $1.91,4.55$ to $6.06 \mathrm{~m} / \mathrm{s}$ with increasing of applied pressure amplitude from $0.03,0.05$ to $0.07 \mathrm{MPa}$, respectively. The droplet volume also increases with $P_{\max }$ from 107,162 to $207 \mathrm{pl}$.

\subsubsection{Influence of $\boldsymbol{P}_{\min }$ on the droplet flying process}

Pictures (d)-(g) in Figs. 9 and 10 illustrate the influence of $P_{\text {min }}$ on the droplet behavior during flying process. Compared with pictures $(\mathrm{d})-(\mathrm{g})$ of baseline case in Fig. 5, the droplet velocity and volume is nearly equal the same. However, a larger $P_{\min }$ causes a shorter tail length of droplet. In Fig. 10 with shortest tail, $P_{\min }=0.04 \mathrm{MPa}$, the satellite droplet combines with primary droplet to form a single droplet during flying process. In Fig. $5, P_{\min }=0.025 \mathrm{MPa}$, the satellite droplet catches up the primary droplet at moment of hitting the substrate. If the $P_{\min }$ is smaller than $0.025 \mathrm{MPa}$, the ink reaches substrate with satellite droplets as shown in Fig. 9 of $P_{\min }=0.01 \mathrm{MPa}$.

\subsubsection{Influence of $\boldsymbol{t}_{\mathrm{o}}$ on the droplet flying process}

Pictures (d)-(g) in Figs. 5, 11 and 12 reveal the variation of droplet during flying process for $t_{\mathrm{o}}=64,48$ and $32 \mu \mathrm{s}$. As the total operating time $t_{\mathrm{o}}$ is reduced from 64 to 48 and $32 \mu \mathrm{s}$, the average droplet velocities decrease accordingly from 4.55 to 4.08 and $3.08 \mathrm{~m} / \mathrm{s}$. The corresponding tail lengths of droplets also decrease. For the case with shortest $t_{\mathrm{o}}$ of $32 \mu \mathrm{s}$, no satellite droplets is found as shown in Fig. 12(d)(g). In the case of $t_{\mathrm{o}}=48 \mu \mathrm{s}$, the satellite droplet would catch up the primary droplet during flying process, and to form a single droplet as shown in Fig. 11(e)-(g). In the case of $t_{\mathrm{o}}=64 \mu \mathrm{s}$, the droplet separates from nozzle exit with longest tail and the satellite droplet forms during flying process as shown in Fig. 5(d)-(f). The satellite and primary droplets will reach the substrate at almost the same time as shown in Fig. 5(g).

\subsubsection{Influence of $\boldsymbol{a}_{\mathrm{p}}$ on the droplet flying process}

Pictures (d)-(g) in Figs. 5, 13 and 14 show the influence of $a_{\mathrm{p}}$ on droplet behavior during flying process for $a_{\mathrm{p}}=1,2$ and $2 / 3$. It is found that the smaller $a_{\mathrm{p}}$, the droplet tail is more unstable. In the case of $a_{\mathrm{p}}=2 / 3$ as shown in Fig. 14(e), the tail breaks to satellites because of capillary instability. The average velocities of the droplets don't change obviously, are $4.55,4.55$ and $4.49 \mathrm{~m} / \mathrm{s}$ for $a_{\mathrm{p}}=2 / 3,1$ and 2 .

\subsection{Evaluation of inkjet printing quality}

The quality of the ejected droplets is closely related to printing quality. In most situations, it is desirable to (1) eliminate satellite droplets, (2) reduce droplet size, and (3) increase droplet velocity. The results in the case 1 , case 4 , case 5 and case 6 are all qualified for criteria (1). For preventing the formation of satellite droplets, the droplet length should be reduced by decreasing the amplitude of the positive pressure (case 1), increasing the amplitude of the negative pressure (case 4) and decreasing the operating period (case 5 and case 6 ).

Figure 15 shows the droplet volume and the average velocity for the baseline and the cases $1-8$. Reducing the droplet volume to improve the pattern resolution could be achieved by decreasing the amplitude of the positive pressure (case 1) and decreasing the operating period (case 5 and case 6). For the criterion (2) consideration, the case 4 is not fitted. The criterion (3) is related to the process throughput.

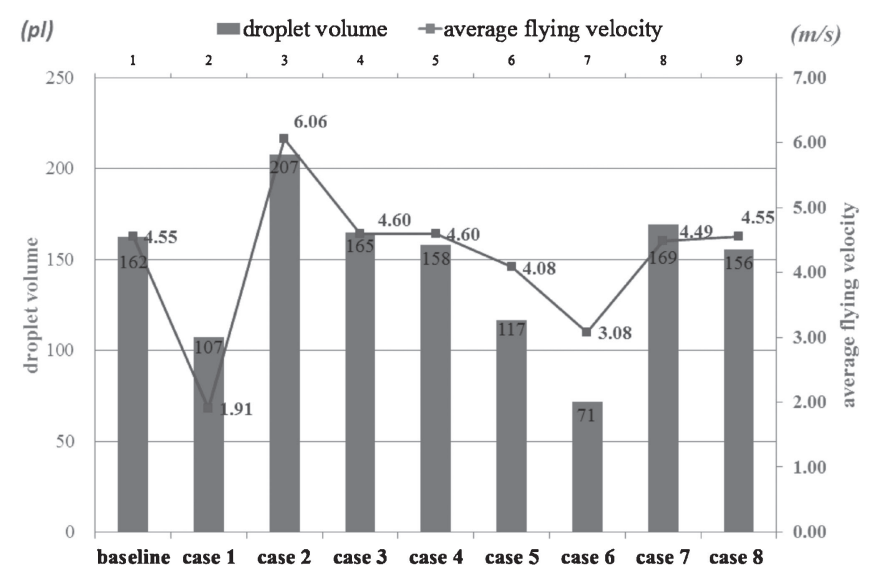

Fig. 15 Droplet volume and average velocity for various cases. 
Generally, the higher droplet velocity could improve the process throughput, but accompanies the larger droplet volume, which decreases the process resolution. Comparing the case 1 with the case 5, both ones possess smaller droplet volume, but only the case 5 can also obtain higher droplet velocity.

\section{Conclusion}

In this study, a fully three-dimension and multi-fluid flows computational system is established to simulate the liquid ejection process in a piezoelectric inkjet print-head. The numerical experiments are performed by replacing pulse voltage with a pressure waveform. The influence of pressure pulse parameters, such as positive pressure amplitude, negative pressure amplitude, operating period and acceleration of pressure on droplet ejection is presented. The major results taken from simulation are summarized as follows:

(1) Increasing the amplitude of the positive pressure $P_{\max }$ could cause the longer tail length, faster droplet velocity and larger droplet volume. The effect of the amplitude of positive pressure on break-up time is not obvious.

(2) Increasing the amplitude of the negative pressure $P_{\min }$ could result in a shorter tail length, shorter breakup time and smaller droplet volume. No apparent difference is observed in the droplet velocity with changes in $P_{\min }$.

(3) The tail length, velocity and volume of droplet would increase with increase of pressure pulse operating time $t_{\mathrm{o}}$.

(4) Increasing the acceleration of the positive pressure $a_{\mathrm{p}}$ would cause the longer tail length, faster droplet velocity and larger droplet volume.

\section{Acknowledgements}

This work has been supported by the National Science Council in Taiwan (NSC97-2221-E-239-009-MY3 and NSC 99-2221-E-131-023), for which the authors are grateful. We also acknowledge the National Center for High-performance Computing for computer time and facilities.

\section{REFERENCES}

1) Q. Liu: J. Mater. Proc. Tech. 115 (2001) 271-283.

2) H. S. Koo, M. Chen and P. C. Pan: Thin Solid Films 515 (2006) 896901.

3) C. N. Hoth, S. A. Choulis, P. Schilinsky and C. J. Brabec: Adv. Mater. 19 (2007) 3973-3978.

4) K. Crowley, E. O’Malley, A. Morrin, M. R. Smyth and A. J. Killard: Analyst 133 (2008) 391-399.

5) M. H. Tsai, W. S. Hwang, H. H. Chou and P. H. Hsieh: Nanotechnology 19 (2008) 335304

6) M. Singh, H. M. Haverinen, P. Dhagat and G. E. Jabbour: Adv. Mater. 21 (2009) 1-13.

7) L. P. Hue: J. Imaging Sci. Tech. 42 (1998) 49-62.

8) N. Bugdayci, D. B. Bogy and F. E. Talk: IBM J. Res. Dev. 27 (1983) 171-180.

9) D. B. Bogy and F. E. Talk: IBM J. Res. Dev. 28 (1984) 314-321.

10) J. E. Fromm: IBM J. Res. Dev. 28 (1984) 323-333.

11) T. M. Liou, K. C. Shih, S. W. Chau and S. C. Chen: Int. Comm. Heat Mass Transfer 29 (2002) 1109-1118.

12) A. S. Yang, J. C. Yang and M. C. Hong: J. Micromech. Microeng. 16 (2006) 180-188.

13) A. K. Sen and J. Darabi: J. Micromech. Microeng. 17 (2007) 14201427.

14) C. S. Kim, S. J. Park, W. Sim, Y. J. Kim and Y. Yoo: Comput. Fluids 38 (2009) 602-612.

15) H. C. Wu, H. J. Lin, Y. C. Kuo and W. S. Hwang: Mater. Trans. 45 (2004) 893-899.

16) H. C. Wu, W. S. Hwang and H. J. Lin: Mater. Sci. Eng. A 373 (2004) 268-278.

17) H. C. Wu, H. J. Lin and W. S. Hwang: Model. Sim. Mater. Sci. Eng. 13 (2005) 17-34.

18) C. W. Hirt and B. D. Nichols: J. Comput. Phys. 39 (1981) 201-225.

19) J. U. Brackbill, D. B. Kothe and C. Zemach: J. Comput. Phys. 100 (1992) 335-354.

20) W. F. Noh and P. R. Woodward: Lecture Notes Phys. 59 (1976) 330.

21) D. L. Youngs: Num. Met. Fluid Dyn. Academic Press (1982) 273285.

22) D. Gueyffier, J. Li, A. Nadim, R. Scardovelli and S. Zaleski: J. Comput. Phys. 152 (1999) 423-456.

23) R. Scardovelli and S. Zaleski: J. Comput. Phys. 164 (2000) 228-237.

24) W. J. Rider and D. B. Kothe: J. Comput. Phys. 141 (1998) 112-152.

25) H. C. Wu, T. R. Shan, W. S. Hwang and H. J. Lin: Mater. Trans. 45 (2004) 1794-1801.

26) H. J. Lin, H. C. Wu, T. R. Shan and W. S. Hwang: Mater. Trans. 47 (2006) 375-382. 